\title{
PREOCUPAÇÃO COM A HONRA NO NORDESTE BRASILEIRO:
} CORRELATOS DEMOGRÁFICOS HONOR CONCERNS IN THE NORTHEAST OF BRAZIL: DEMOGRAPHIC CORRELATES

\author{
Valdiney V. Gouveia \\ Universidade Federal da Paraíba, João Pessoa/PB, Brasil \\ Valeschka M. Guerra \\ Universidade Federal do Espirito Santo, Vitória/ES, Brasil \\ Rafaella de Carvalho Rodrigues Araújo \\ Universidade Federal da Paraíba, João Pessoa/PB, Brasil
}

Lilian Kelly Sousa Galvão

Universidade Federal de Campina Grande, Campina Grande /PB, Brasil

Sandra Souza da Silva

Universidade Federal da Paraíba, João Pessoa/PB, Brasil

\section{RESUMO}

Esta pesquisa pretendeu conhecer os correlatos demográficos da preocupação com a honra, considerando três grupos religiosos (católicos, batistas e espíritas) em duas cidades do Nordeste brasileiro (João Pessoa e Recife). Dois estudos foram realizados. No Estudo 1, participaram 312 pessoas, contatadas em igrejas e templos, que apresentaram média de idade de 32 anos $(d p=15,28)$, sendo a maioria mulheres $(63 \%)$. No Estudo 2, participaram 178 pessoas da população geral, a maioria mulheres $(69 \%)$, com média de idade de 32 anos ( $d p$ $=10,51)$. Os participantes responderam à Escala de Preocupação com a Honra e questões demográficas. Os resultados indicaram que a honra social e familiar foram consideradas mais importantes. Os principais correlatos da preocupação com a honra em ambos os estudos foram a idade e a religião dos participantes. A importância da religião na preocupação com a honra é discutida, assim como limitações e propostas para pesquisas futuras.

Palavras-chave: honra; demográfica; religião; idade; gênero.

\begin{abstract}
The present research aimed at investigating the demographic correlates of honor concerns in three religious groups (Catholics, Baptists, and Spiritists) in two cities in northeastern Brazil (João Pessoa and Recife). Two studies were conducted to achieve this goal. In Study 1, participants were 312 people from general population, contacted in churches and temples. They presented a mean age of 32 years $(s d=15.28)$ and were mostly women (63\%). In Study 2, participants were 178 people from general population, mostly women (69\%), with a mean age of 32 years $(s d=10.51)$. Participants answered the Honour Concerns Scale and demographic questions. Results indicated a higher endorsement to social and family honor. The main correlates of honor concerns across studies were age and participants' religious denomination. The importance of religion for honor concerns' endorsement is discussed, as well as studies limitations and further research.
\end{abstract}

Keywords: honour; demographic; religion; age; gender.

\section{Introdução}

"A reputação de mil anos pode ser determinada pela conduta de uma hora." (Provérbio tradicional japonês)

Provérbios são ditos populares, tradicionalmente de caráter oral, que capturam pontos de vista comuns em uma dada cultura. Menandro, Rolke e Bertollo (2005) afirmam que os provérbios tornam-se parte das tradições de uma sociedade, expressando normas sociais e ensinamentos morais. Provérbios sobre a honra podem ser encontrados em diversas culturas. Por exemplo, no Vietnã se diz que "é melhor morrer do que viver com uma má reputação"; na África, fala-se que 
"onde não há vergonha, não há honra"; na Índia, diz-se que "cada homem é o guardião da sua honra"; e, no Brasil, é comum encontrar o provérbio de que "honra se lava com sangue" (Baer, 2001).

Segundo Arthur Schopenhauer, filósofo alemão, em sua obra "A arte de se fazer respeitar, ou Tratado sobre honra", esta pode ser definida como a opinião de outras pessoas acerca do valor de um indivíduo e, aos olhos deste, seria o medo dessa opinião (Schopenhauer, 2003). Em uma definição mais formal, segundo o Dicionário Houaiss da Língua Portuguesa (Houaiss, s.d.), honra pode ser compreendida como um "princípio ético que leva alguém a ter uma conduta virtuosa, corajosa, e que lhe permite gozar de bom conceito junto à sociedade".

Para Fischer, Manstead e Rodriguez-Mosquera (1999), o termo honra pode ser considerado, na psicologia, como sendo tanto um atributo pessoal, quando descreve a autoestima e a reputação de um indivíduo, mas também como um atributo coletivo, pois é compartilhado com seu grupo social ou familiar (ver também Pitt-Rivers, 1965).

Wikan (1984) propõe que o conceito de honra de um indivíduo está associado à sua concepção de moral; assim, ter honra significa ter caráter, força moral. Ameaças ou desrespeitos à honra de um indivíduo ou de seu grupo tendem a despertar reações ou emoções negativas fortes, tais como raiva e vergonha (Rodriguez-Mosquera, Manstead, \& Fischer, 2002a). Em função desta importância atribuída à reputação e sua associação com reações emocionais, agressões e crimes podem ser cometidos e justificados em sua defesa (Souza, 2010). A exemplo disso, Shackelford (2005) argumenta que um dos elementos chave de locais onde predomina a cultura de honra é que as pessoas, principalmente os homens, estão preparados para utilizarem força física e violência, se necessário, para defender sua própria reputação e a de sua família.

Vandello e Cohen (2003) sugerem que o Brasil apresenta características de uma cultura de honra. Estes autores observaram uma tendência maior de brasileiros a perceber um homem traído como menos honrado e menos masculino, quando comparados com estadunidenses. Além disso, brasileiros apresentaram maior tendência a aceitar a violência de um marido em casos em que a esposa foi infiel.

Neste sentido, Vellasco (2005) apresenta uma análise de documentação judicial na Comarca do Rio das Mortes, em Minas Gerais, durante o século XIX. $\mathrm{Na}$ oportunidade, sugere que nesta comunidade a violência era considerada uma expressão legítima de valores sociais, como honra e dignidade.
Apesar de o Brasil ter sido identificado como um país que possui tendências claras de preocupação acentuada com a honra (Rohden, 2001; Vellasco, 2005), constata-se uma lacuna na literatura psicológica neste país quanto a estudos a respeito da temática honra. Quando encontrados, percebe-se que estes têm sido levados a cabo, principalmente, nas áreas antropológica e jurídica (Sigaud, 2004; Vellasco, 2005). Corroborando este aspecto, em busca realizada no Index Psi (2010), foram encontrados apenas três artigos sobre honra: dois deles enfocavam narrativas psicanalistas sobre a vergonha (Ferrari, 2007; Verztman, 2005) e o terceiro centrava-se no estudo da humilhação (Alencar \& La Taille, 2007). Não foram encontrados no contexto brasileiro quaisquer estudos acerca da associação entre a preocupação com a honra e construtos presumivelmente relevantes para explicála (e.g., valores humanos, religiosidade) ou em que se conhecessem seus correlatos demográficos.

\section{Preocupação com a honra}

De acordo com Rodriguez-Mosquera, Manstead e Fischer (2002b), as culturas de honra seguem um conjunto de valores que estabelecem padrões normativos para os comportamentos de seus integrantes. Estes valores são representados em dimensões, segundo seus conteúdos (RodriguezMosquera, Fischer, \& Manstead, 2004), configurando quatro tipos principais de preocupações com a honra (Rodriguez-Mosquera et al., 2002a, 2002b):

Honra familiar. A preocupação com a honra da família indica que a reputação individual é interdependente ou compartilhada com a família. Esta interdependência baseia-se na identidade que comungam os indivíduos que compõem a família. Nesse sentido, comportamentos de membros individuais têm um impacto na reputação de cada indivíduo e da família como um todo. Esta preocupação enfatiza a importância de respeitar o "nome" da família. A honra familiar, desse modo, pode ser interpretada como o valor e o status que a família possui aos olhos dos outros.

Honra social. Previamente denominada como integridade ou interdependência social, essa dimensão expressa uma interdependência do self com grupos sociais mais amplos, envolvendo a reputação do indivíduo em relacionamentos interpessoais. Há uma ênfase na necessidade de estabelecer e aprofundar a harmonia nas relações sociais mediante a manutenção da integridade. Esta é associada com a percepção internalizada da honra, que garante o respeito dos demais, enfatizando-se valores como generosidade, 
honestidade e lealdade, não apenas aos outros, mas como base dos próprios princípios individuais.

Honra masculina. Expressa a necessidade de se mostrar forte e capaz de responder a ofensas que possam diminuir a masculinidade, a honra do próprio indivíduo ou de sua família. Destaca a virilidade e a associação desta com a responsabilidade de manter a autoridade masculina e defender sua família. Comumente indica-se que quem um homem é está interligado com o "quão homem" ele é (Nisbett \& Cohen, 1996). Portanto, essa condição é definida em termos de força e respeito. Entretanto, tal preocupação não é exclusiva dos homens, as mulheres também são uma parte essencial deste padrão normativo, ensinando-o a seus filhos e reforçando-o quando apresentado por parte de seus parceiros e familiares.

Honra feminina. Expressa a necessidade de manter a castidade sexual, fortemente associada à honra familiar. Assim, o comportamento de uma mulher pode trazer a desgraça para a reputação da família como um todo. Não se espera, no entanto, que as mulheres defendam ativamente o nome da família, como ocorre com os homens. Esperase apenas que elas o protejam, agindo de forma apropriada (Werbner, 2007).

Em resumo, os dois primeiros tipos de honra são importantes para qualquer indivíduo que faz parte de uma cultura de honra. No entanto, como descritos, também são encontrados códigos de honra específicos de gênero, que enfatizam o que é ser um homem e uma mulher nessas culturas. A preocupação com a honra feminina está associada com aquela referente à honra masculina, pois também é esperado que os homens protejam a honra feminina. Nesse sentido, consequências comuns de condutas sexuais impróprias, por parte da mulher, que ameacem a honra masculina e da família tendem a suscitar e "justificar" a agressão e a violência (Nisbett \& Cohen, 1996; RodriguezMosquera et al., 2002b).

Fica evidente, portanto, que a honra é um construto que pode ser central na explicação de diversos comportamentos em culturas de honra, sobretudo aqueles que ameaçam as pessoas e suas relações interpessoais quando os padrões "morais" estabelecidos não são cumpridos (Souza, 2010). Nesse caso, nas culturas de honra, a percepção de que tais padrões possam estar sob ameaça externa, sem punição ou representação adequada de meios de segurança, como os governamentais, faz com que se desenvolvam comportamentos característicos nos moradores locais, como os agressivos e de autoproteção (Shackelford, 2005). Desse modo, justifica-se o interesse em compreender a honra, identificando seus correlatos. Precisamente este foi o aspecto que motivou o presente estudo, orientado a identificar correlatos demográficos da preocupação com a honra em um contexto cultural eminentemente tradicional e coletivista, como o Nordeste brasileiro (Gouveia, Albuquerque, Clemente, \& Espinosa, 2002; Hofstede, Hilal, Malvezzi, Tanure, \& Vinken, 2010).

Para Nisbett e Cohen (1996), o contexto socioeconômico e demográfico é um dos propiciadores de uma cultura de honra. Os autores propõem que esse tipo de cultura é mais fortemente estabelecido em sociedades pastoris quando se compara com sociedades agrícolas ou coletoras. Nestas sociedades, o uso de ameaças ou violência física real é maior devido às constantes ameaças de perda / roubo de seus animais pela ação de outros. A escassez causada pela ação predatória de terceiros gera a necessidade de um indivíduo defender suas posses mediante o uso de violência, especialmente em sociedades em que há pouca confiança na segurança pública (ver também Shackelford, 2005).

\section{Correlatos demográficos}

Diversos atributos sociais e demográficos podem ser investigados como fonte de influência em construtos psicológicos, tais como sexo, região geográfica, religião e renda (Gouveia \& Clemente, 2000). Tais informações, coletadas a nível individual, podem ser associadas a outras no nível cultural de análise, permitindo comparações denominadas transculturais acerca de padrões de atitudes, valores ou comportamentos observados (Gouveia \& Ros, 2000). Não obstante, o presente estudo limita-se ao nível individual de análise, procurando conhecer a influência de três variáveis demográficas principais na preocupação com a honra, como seguem:

$\underline{\text { Sexo. }}$ Pesquisas sugerem que as mulheres tendem a atribuir maior importância ao coletivismo do que os homens (Gouveia \& Clemente, 2000). Schwartz e Rubel (2005), por exemplo, apontam diferenças de gênero com relação aos valores humanos, indicando que as mulheres atribuem consistentemente maior importância à benevolência (preocupar-se com o bem estar daqueles com os quais se tem contato frequente) e ao universalismo (tentar compreender e preocupar-se com o bem estar da natureza e pessoas em geral), enquanto os homens o fazem em relação aos valores poder (ter controle ou domínio sobre recursos e pessoas), hedonismo (sentir prazer e satisfação por si mesmo), autorrealização (obter sucesso pessoal e demonstrar competência de acordo com o esperado socialmente) e autodireção 
(ter pensamentos e ações independentes). No que diz respeito à preocupação com a honra, RodriguezMosquera et al. (2002a) observaram que as mulheres atribuíram maior importância à honra feminina quando comparadas com os homens. As mulheres apresentaram maior preocupação com a opinião de outras pessoas, assim como reações emocionais mais intensas de vergonha e raiva em situações associadas com o questionamento de sua reputação.

Idade. Esta é considerada uma das variáveis mais relevantes para explicar valores e tendências de comportamento mais tradicionais (Schwartz $\&$ Rubel, 2005). Gouveia e Clemente (2000), por exemplo, apontam que pessoas mais velhas atribuem maior importância a uma orientação coletivista quando comparadas às mais jovens. Truett (1993), estudando a influência da idade no conservadorismo, sugere que mesmo participantes de diferentes gêneros, níveis educacionais e localizações geográficas apresentam um maior conservadorismo com o aumento da idade.

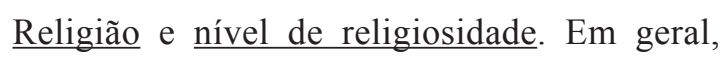
percebe-se que quanto maior a religiosidade do participante, maior a importância atribuída ao coletivismo (Gouveia \& Clemente, 2000). No entanto, não se encontrou qualquer estudo que relacionasse os diferentes grupos religiosos com a importância dada à preocupação com a honra. Contudo, além da pertença às diferentes denominações, Gouveia e Clemente (2000) sugerem que a importância atribuída à religião pelo participante está associada a um maior coletivismo. Comunidades religiosas, em geral, desenvolvem códigos que devem ser seguidos por todos os seus membros como um guia central, abrangendo desde expressões externas de sua religiosidade (e.g., acessórios e vestimentas), normas comportamentais (e.g., qual a conduta apropriada, que alimentos são considerados adequados) até tipos de valores que devem ser transmitidos e enfatizados no grupo (e.g., obediência aos pais e sacerdotes, respeito às tradições) (Cohen \& Hill, 2007). A importância que a população brasileira atribui à religião e à religiosidade (Bucher, 2007) sugere que deva ser considerada com respeito aos padrões normativos expressados por meio da preocupação com a honra.

Em resumo, a preocupação com a honra pode apresentar relação com diversos antecedentes demográficos. Conhecer o padrão de associação e o poder explicativo dessas variáveis poderá contribuir para a compreensão do papel de diferenças individuais e culturais na preocupação com a honra. Com tal propósito, dois estudos foram realizados.

\section{Estudo 1. Correlatos demográficos da preocupação com a honra}

Este estudo procurou reunir as primeiras evidências acerca da contribuição de variáveis demográficas para explicar diferentes tipos de preocupação com a honra no contexto do Nordeste brasileiro. Procurou ainda avaliar o tipo de preocupação com honra que é mais característico das pessoas que fizeram parte desta pesquisa.

\section{Definição operacional das variáveis}

Considerou-se como a variável critério a preocupação com a honra, correspondendo às pontuações dos participantes no instrumento correspondente; no caso das variáveis antecedentes, foram as três demográficas anteriormente tratadas: idade (quantos anos a pessoa tinha à época da coleta de dados), sexo (definida como dummy, assumindo os valores $0=$ Feminino, $1=\underline{\text { Masculino) }}$ e religião. Levaram-se em conta dois indicadores: (1) tipo de religião, considerando-se participantes de três orientações: Católica, Protestante (Batista) e Espírita. As respostas foram tabuladas para cada religião como "não" e "sim", correspondendo a variáveis dummy com os valores 0 e 1, respectivamente; e (2) nível de religiosidade, respondida pelos participantes em termos do quanto se percebiam como religiosos, empregandose escala de resposta de cinco pontos, variando de $1=$

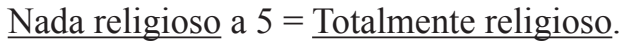

\section{Participantes}

Participaram 312 pessoas de João Pessoa (PB) e Recife (PE). A maioria era do sexo feminino (63\%), com idades variando de 13 a 88 anos $(\underline{m}=31,9 ; \underline{d p}=$ $15,28)$. Com relação à religião, a maior parte indicou ser católica (46\%), seguida por evangélicos (batistas) (35\%) e, por fim, espíritas (19\%). Estes participantes apresentaram religiosidade média autopercebida de $4,1(\underline{\mathrm{p}}=0,84)$, valor superior à mediana teórica $(3) \mathrm{da}$ escala de resposta [ $\mathrm{t}(301)=21,74, \mathrm{p}<0,001]$.

\section{Instrumentos}

Os participantes foram solicitados a responder um livreto composto por três partes, duas das quais são de interesse principal neste estudo: (1) Escala de Preocupação com a Honra (EPH). Elaborada originalmente em língua espanhola, reunindo evidências de validade de construto (consistência interna) na Espanha e Holanda (Rodriguez-Mosquera, Manstead, \& Fischer, 2000), esta medida conta com 
25 itens divididos em quatro subescalas: honra familiar (e.g., sua família tivesse má reputação), honra social (e.g., você traísse outras pessoas), honra masculina (e.g., você não se defendesse quando os outros o insultassem) e honra feminina (e.g., você tivesse relações sexuais antes do casamento). Com o fim de respondê-los, o participante deve indicar em que medida se sentiria mal em cada contexto descrito, empregando escala de resposta de nove pontos, variando de 1 (Nem um pouco mal) a 9 (Muito mal). Em estudo realizado no Brasil por Guerra, Gouveia, Araújo, Andrade e Bezerra (no prelo), também se constatou claramente uma estrutura tetrafatorial, coerente com esta proposta inicial. Os indicadores de ajuste deste modelo foram adequados, além de todos os índices de consistência interna dos fatores (alfa de Cronbach) terem sido superiores a 0,60. Resultados positivos também foram encontrados na análise de confiabilidade composta (CC) dos mesmos. Com exceção do fator honra masculina que se situou em 0,64 ou mais, os demais superaram 0,70 . Nesse sentido, podem-se assegurar os parâmetros psicométricos da EPH também no Brasil; e (2) perguntas demográficas. Foram incluídas as seguintes variáveis: idade, sexo, religião e nível de religiosidade, como anteriormente descritas.

\section{Procedimento}

Os pesquisadores entraram em contato com os responsáveis de igrejas e templos religiosos escolhidos por conveniência, considerando a disponibilidade manifesta e/ou os contatos prévios realizados com seus representantes. Após o consentimento destes, com o auxílio de bolsistas previamente treinadas, procedeuse à aplicação dos instrumentos. Na oportunidade, os pesquisadores se apresentavam, indicavam seu interesse de conhecer formas que as pessoas têm de pensar e agir no seu dia a dia, solicitando em seguida a participação voluntária daqueles que estavam frequentando igrejas ou templos por ocasião da coleta. A todos foi dito que as respostas deveriam ser individuais, garantindo-se o anonimato. Foram dadas oralmente instruções gerais sobre como preencher os questionários, permanecendo os pesquisadores no ambiente de coleta com o fim de esclarecer as dúvidas eventuais. O tempo médio para concluir sua participação foi de aproximadamente 20 minutos.

\section{Análise de dados}

O PASW (versão 18) foi utilizado para tabular e realizar as análises dos dados. Calcularam-se estatísticas descritivas (medidas de tendência central e dispersão, distribuição de frequência), coeficientes de correlação ( $r$ de Pearson), procurando, neste caso, investigar a relação entre as dimensões da preocupação com a honra e as variáveis demográficas, teste t para medidas repetidas com o fim de saber o nível de religiosidade dos participantes e Manova para medidas repetidas a fim de avaliar a maior preocupação com a honra no grupo pesquisado.

\section{Resultados e discussão}

Inicialmente, procurou-se conhecer as pontuações dos participantes nos fatores da medida de honra, avaliando se haveria diferenças entre elas. Foi realizada uma análise de variância mista com design 2 (Sexo: masculino, feminino) x 4 (Honra: família, social, masculina, feminina). Os resultados indicaram um efeito principal da honra, Lambda de Wilks = $0,20, \mathrm{~F}(3,307)=408,37, \mathrm{p}<0,001, \eta^{2}$ parcial $=0,80$. Especificamente, o teste post hoc de Bonferroni indicou que todos diferiam entre si $(\mathrm{p}<0,05)$, como seguem: honra social $(\mathrm{m}=8,0, \mathrm{dp}=0,95)$; honra familiar $(\mathrm{m}=$ $7,6, \mathrm{dp}=1,33)$; honra feminina $(\mathrm{m}=6,3, \mathrm{dp}=1,84)$; e honra masculina $(\mathrm{m}=5,9, \mathrm{dp}=1,14)$. Portanto, os participantes enfatizam mais a preocupação com a honra social e honra familiar, fazendo-o em menor medida com relação à honra masculina. Possivelmente, tal padrão de preocupação indica a cultura em que vivem as pessoas do estudo, que é eminentemente coletivista, priorizando preocupações mais com respeito ao seu grupo de pertencia e sua família do que aquelas mais pessoais (Gouveia et al., 2002; Hofstede et al., 2010).

Foi observado um efeito principal do sexo apenas marginal, não significativo, $F(1,309)=3,23$, $\mathrm{p}=0,07, \eta^{2}$ parcial $=0,01$. No entanto, a interação sexo $\mathrm{x}$ honra foi significativa, $\mathrm{F}(3,927)=19,76, \eta^{2}$ parcial $=0,06$. Esta análise indicou que as mulheres apresentaram pontuações similares no que diz respeito à honra da família $(\mathrm{m}=7,63)$ e honra social $(\mathrm{m}=8,05)$ quando comparadas com as pontuações dos homens (honra da família $\mathrm{m}=7,55$; honra social $\mathrm{m}=7,98$ ). No entanto, os homens apresentam maiores pontuações de honra masculina $(\mathrm{m}=6,04)$ do que as mulheres $(m=5,76)$, e estas apresentam maiores pontuações de honra feminina $(\mathrm{m}=6,66)$ do que os homens $(m=5,69)$. Estes resultados estão de acordo com achados descritos previamente na literatura (Rodriguez-Mosquera et al., 2002b).

Posteriormente, foram calculadas as correlações entre as dimensões de honra e destas com as variáveis demográficas, sendo os resultados apresentados na Tabela 1. Como é possível observar nesta tabela, participantes que endossam qualquer tipo de honra tendem a considerar importantes os outros três tipos de 
honra (social, masculina e feminina). Esta associação entre as dimensões da honra reforçam a existência deste código cultural de conduta, que perpassa as relações interpessoais de um indivíduo.

Além disso, uma maior preocupação com a honra masculina apresentou correlação direta com a idade dos participantes, sugerindo que quanto maior a idade, maior é a preocupação dos indivíduos com esse tipo de honra. Isso talvez seja indicação de maior conservadorismo das pessoas mais velhas (Schwartz \& Rubel, 2005; Truett, 1993), apegandose à honra masculina como forma de reproduzir papéis tradicionais em cultura coletivista (Gouveia \& Clemente, 2000).

Tabela 1. Estudo 1 - Correlatos demográficos da preocupação com a honra

\begin{tabular}{lcccc}
\hline & \multicolumn{4}{c}{ Preocupação com a honra } \\
\cline { 2 - 5 } Variáveis & Honra familiar & Honra social & Honra masculina & Honra feminina \\
\hline Honra familiar & - & & \\
Honra social & $0,57^{* *}$ & - & - & \\
Honra masculina & $0,54^{* *}$ & $0,37^{* *}$ & $0,29^{* *}$ & - \\
Honra feminina & $0,48^{* *}$ & $0,43^{* *}$ & $0,27^{* *}$ & 0,03 \\
Idade & 0,08 & 0,09 & $0,10^{\dagger}$ & $0,33^{* *}$ \\
Batista & 0,06 & 0,09 & 0,00 & $-0,08$ \\
Católica & $0,20^{* *}$ & $-0,01$ & $-0,12^{*}$ & $-0,30^{* *}$ \\
Espírita & $-0,33^{* *}$ & $-0,10^{\dagger}$ & $-0,02$ & $0,11^{\dagger}$ \\
Religiosidade & 0,08 & 0,08 & & \\
\hline
\end{tabular}

Nota. $\cdots \mathrm{p}<0,10 ; * \mathrm{p}<0,05 ; * * \mathrm{p}<0,01$.

Os participantes que afirmaram ser de denominação evangélica (batista) foram marginalmente mais prováveis a endossar a honra masculina, mas foram principalmente enfáticos em atribuir maior importância à honra feminina; por outro lado, aqueles indivíduos que indicaram ser de denominação católica atribuíram maior importância à honra familiar. No caso dos participantes espíritas, eles não endossaram diretamente quaisquer dos tipos de honra, porém discordaram significativamente das dimensões de honra familiar, masculina e feminina, além de apresentarem uma relação marginal, também negativa, com a honra social. O nível de religiosidade dos participantes apresentou apenas uma relação marginal direta com a honra feminina. Embora se estimasse o papel da religião e religiosidade como correlatos dos tipos de honra, não existiam evidências prévias que permitissem conhecer a direção. Nesse sentido, estes resultados precisam ser tomados como heurísticos, demandando checar sua estabilidade.
Em resumo, o presente estudo evidenciou a maior preocupação das pessoas neste contexto de pesquisa com dimensões da honra que enfocam o contexto social, a família. As variáveis demográficas tiveram papel destacável como correlatos da preocupação com os diversos tipos de honra, destacando-se os tipos de honra masculina e feminina. Resta saber em que medida estes achados são corroborados, o que motivou a realização de um segundo estudo.

\section{Estudo 2. Replicando evidências dos correlatos da preocupação com a honra}

Este estudo teve como objetivo principal replicar os achados do estudo anterior, considerando uma amostra independente de participantes. Foram consideradas as mesmas variáveis e definições respectivas, como previamente apresentadas. 


\section{Participantes}

Participaram 178 pessoas da população geral de João Pessoa (PB). A maioria era do sexo feminino (69\%), com idades variando de 16 a 69 anos $(\mathrm{m}=31,9$; $\mathrm{dp}=10,51)$. Com relação à religião, majoritariamente tais pessoas se denominaram batistas (44\%), seguidas das que disseram ser católicas $(35 \%)$ e, por fim, espíritas $(20 \%)$. O conjunto dos participantes revelou tendência moderada à religiosidade, com média de 3,7 $[\mathrm{dp}=1,03 ; \mathrm{t}(134)=7,81, \mathrm{p}<0,001]$.

\section{Instrumentos e procedimento}

Os participantes foram solicitados a responder um questionário formado pelos mesmos instrumentos do Estudo 1: Escala de Preocupação com a Honra $(\mathrm{EPH})$ e questionário demográfico. Seguiu-se o mesmo procedimento descrito naquele estudo. Os dados foram tabulados e analisados por meio do PASW (versão 18), recorrendo-se às mesmas estatísticas. Entretanto, como o fim de avaliar a replicabilidade dos coeficientes de correlação para os tipos de honra e variáveis demográficas neste estudo em relação ao anterior, empregou-se o programa Corrs, desenvolvido em Excel (Brannick, 2007).

\section{Resultados e discussão}

Da mesma forma que no Estudo 1, uma análise de variância mista com design 2 (Sexo: masculino, feminino) x 4 (Honra: família, social, masculina, feminina) foi conduzida. Foi observado o efeito principal do tipo de honra, Lambda de Wilks $=0,20$, $\mathrm{F}(3,134)=183,54, \mathrm{p}<0,001, \eta^{2}$ parcial $\left.=0,80\right]$, evidenciadas, como seguem, de acordo com o teste post hoc de Bonferroni ( $\mathrm{p}<0,05)$ : honra social $(\mathrm{m}=$ $8,2, \mathrm{dp}=0,82)$; honra familiar $(\mathrm{m}=7,3, \mathrm{dp}=1,39)$; honra masculina $(\mathrm{m}=5,9, \mathrm{dp}=1,24)$; e honra feminina $(\mathrm{m}=5,8, \mathrm{dp}=1,92)$. Corroborando o Estudo 1, os participantes apresentaram maior preocupação com as honras social e familiar, sendo esta menos evidente no que se refere às honras masculina e feminina, que, no presente estudo, não se diferenciaram entre si. Estes achados, provavelmente, suportam a explicação de que tais preocupações podem ser um reflexo da cultura coletivista destas pessoas, que enfatizam elementos mais sociais de preocupação (Hofstede et al., 2010).

Diferentemente do estudo anterior, não foram encontrados o efeito principal do sexo, $\mathrm{F}(1,136)=$ $0,01, p=0,92$, ou a interação desta variável com o tipo de honra, $\mathrm{F}(3,408)=1,23, \mathrm{p}=0,29$.

Quanto aos correlatos demográficos dos tipos de honra, os resultados são mostrados na Tabela 2 a seguir. A honra feminina apresentou magnitude de correlação diferente do estudo anterior com relação às variáveis de religião batista $(\mathrm{z}=2,61, \mathrm{p}<0,01)$, católica $(\mathrm{z}=4,88, \mathrm{p}<0,001)$ e espírita $(\mathrm{z}=2,53, \mathrm{p}$ $<0,01)$, além de religiosidade $(\mathrm{z}=1,76, \mathrm{p}<0,05)$. Destaca-se, entretanto, que as direções das correlações foram idênticas, sendo uma única inferior neste estudo: religião batista.

Tabela 2. Estudo 2 - Correlatos demográficos da preocupação com a honra

\begin{tabular}{|lcccc|}
\hline & \multicolumn{4}{c|}{ Preocupação com a honra } \\
\cline { 2 - 5 } Variáveis & Honra familiar & Honra social & Honra masculina & Honra feminina \\
\hline Honra familiar & - & & & \\
Honra social & $0,35^{* *}$ & - & & \\
Honra masculina & $0,65^{* *}$ & $0,24^{* *}$ & - & - \\
Honra feminina & $0,41^{* *}$ & $0,35^{* *}$ & $0,29 * *$ & $-0,05$ \\
Idade & $0,23^{* *}$ & $0,14^{+}$ & $0,39^{* *}$ & $0,53 * *$ \\
Batista & $0,27^{* *}$ & $-0,04$ & 0,12 & $-0,49 * *$ \\
Católica & $-0,09$ & $-0,03$ & 0,02 & $-0,07$ \\
Espírita & $-0,23^{* *}$ & 0,09 & $-0,17^{*}$ & $0,27 * *$ \\
Religiosidade & $-0,07$ & $0,16^{+}$ & $-0,09$ & \\
\hline Nota. $\cdots \mathrm{p}<0,10 ; * \mathrm{p}<0,05 ; * * \mathrm{p}<0,01$. & & & \\
\hline
\end{tabular}


A religião espírita apresentou no presente estudo correlação com honra social contrária àquela descrita previamente $(\mathrm{z}=2,01, \mathrm{p}<0,05)$. Neste caso, pessoas que se indicaram espíritas foram mais propícias a se preocuparem com este tipo de honra. A honra familiar também apresentou resultado diferente neste estudo em relação às religiões batista $(z=2,29, p<0,05) \mathrm{e}$ católica $(\mathrm{z}=3,10, \mathrm{p}<0,001)$; no primeiro caso variou apenas a magnitude do coeficiente de correlação, que aumentou, mas no segundo mudou a direção do coeficiente, que passou de positivo para negativo, embora este último não tenha sido significativo. As correlações da variável idade com os tipos de honra se mantiveram invariantes.

Parece evidente, considerando os resultados anteriormente descritos, que a religiosidade e, principalmente, as religiões, possuem um papel complexo como correlatos dos tipos de honra, não apresentando resultados estáveis. Como asseveram Gouveia e Clemente (2000), esta é uma variável importante para explicar a orientação coletivista, sendo comprovado seu papel também em relação à honra. Contudo, as magnitudes de correlações são variáveis, o sendo também algumas vezes a direção. Isso pode dever-se ao menos a dois aspectos: (1) a forma como foi definida a religião, tratada como variável dummy, restringindo a escolha que o respondente fazia entre as alternativas apresentadas; e (2) a diversificação das religiões no Brasil, reunindo elementos e tendências muito diversas (Jacob, 2004), o que pode resultar em um padrão complexo, não muito nítido. Estes aspectos demandarão maior atenção acerca da influência das religiões na preocupação com a honra, talvez elaborando indicadores complementares que atestem o tipo de religião da pessoa.

Complementando as análises acerca da associação entre as dimensões de honra e seus correlatos demográficos, realizaram-se análises de regressão linear múltipla, introduzindo como variável critério cada dimensão de honra e como variáveis antecedentes as demográficas que apresentaram correlação significativa com a dimensão analisada. Os resultados são mostrados na Tabela 3 .

Tabela 3. Preditores da preocupação com a honra

\begin{tabular}{llcccc}
\hline $\begin{array}{l}\text { Preocupação com } \\
\text { a honra }\end{array}$ & Preditores & $\mathrm{R}_{\text {ajustado }}^{2}$ & $\mathrm{~F}_{\text {mudança }}$ & $\beta$ & $\mathrm{t}$ \\
\hline Honra familiar & Batista & 0,70 & $10,75^{* *}$ & 0,34 & $4,23^{* *}$ \\
& Idade & 0,15 & $14,45^{* *}$ & 0,31 & $3,80^{* *}$ \\
Honra social & - & - & - & - & - \\
Honra masculina & Idade & 0,14 & $24,09^{* *}$ & 0,43 & $5,58^{* *}$ \\
& Espírita & 0,20 & $10,23^{* *}$ & $-0,25$ & $3,20^{* *}$ \\
Honra feminina & Batista & 0,30 & $59,23 * *$ & 0,39 & $4,21^{* *}$ \\
& Católica & 0,33 & $7,01 * *$ & $-0,25$ & $2,65^{* *}$ \\
\hline
\end{tabular}

Nota. ${ }^{*} p<0,05 ; * * p<0,01$.

De acordo com esta tabela, no que diz respeito à honra familiar, unicamente as variáveis tipo de religião (batista) e idade dos participantes a predisseram. A honra social não foi predita por quaisquer das variáveis demográficas. No caso da honra masculina, seus preditores demográficos foram a idade (uma relação direta) e a denominação espírita (uma relação inversa). Finalmente, a preocupação com a honra feminina foi explicada exclusivamente pelas denominações religiosas batista (diretamente) e católica (inversamente).

Portanto, como anteriormente discutido, a religião é uma variável importante neste contexto, restando compreender melhor seu papel. 


\section{Considerações finais}

As variáveis demográficas são geralmente consideradas como antecedentes em análises estatísticas de construtos psicológicos (Gouveia \& Clemente, 2000). O presente estudo representa um esforço no sentido de estimar a contribuição de algumas destas variáveis para explicar a variabilidade na preocupação com diferentes dimensões de honra no contexto brasileiro, especificamente em duas cidades do Nordeste (João Pessoa e Recife), que pode ser entendido como uma cultura coletivista (Hofstede et al., 2010) e de honra (Souza, 2010). Confia-se que os objetivos dos estudos previamente descritos tenham sido alcançados, embora seja necessário destacar suas limitações potenciais.

Quanto a tais limitações, consideraram-se participantes unicamente de duas cidades do Nordeste, podendo não retratar sua diversidade; a religião foi considerada de forma simples, considerando apenas a escolha dos participantes de uma lista apresentada; e foi assumido que o contexto de pesquisa é coletivista, enfocando a preocupação com a honra. Além disso, não foram consideradas outras variáveis socioeconômicas importantes, tais como a classe social, a renda ou o contexto urbano ou rural, que podem influenciar na importância da honra para os indivíduos. É necessário que pesquisas futuras sobre a honra levem em consideração tais indicadores.

No entanto, tais limitações não invalidam os resultados encontrados nos estudos descritos, cuja principal contribuição foi apresentar a importância da associação da honra com a religião. Para Cohen e Hill (2007), a religião é um sistema de crenças que pode ser considerado e investigado como uma cultura. Em geral, enfatiza-se não apenas obediência às autoridades religiosas, mas também a outros valores morais, tais como respeito pela família, justiça social e igualdade (Geyer \& Baumeister, 2005). Em um país no qual a maioria da população indica ser católica (Jacob, 2004), mas onde há uma redução na participação ativa nesta comunidade religiosa (Negrão, 2008), conhecer as crenças e práticas religiosas das pessoas pode ajudar a compreender as divergências observadas.

No entanto, apesar de tais divergências, alguns padrões podem ser evidenciados nos resultados. O primeiro deles é a importância atribuída por participantes de denominação batista à honra familiar eà honra feminina, observado nos dois estudos. Pesquisas sociológicas indicam que grupos protestantes, como os batistas, atribuem maior importância a papéis tradicionais de gênero (Sherkat \& Ellison, 1999), o que justificaria tais resultados.

O segundo padrão a ser observado é a importância da honra masculina para participantes de maior idade. Esta dimensão da honra enfatiza, especificamente, o papel tradicional masculino e sua importância no comando da família (Rodriguez-Mosquera et al., 2002a), o que está de acordo com uma visão mais conservadora dos papéis sociais. Gerações anteriores em um contexto social tradicional e coletivista, como é o nordeste brasileiro, podem apresentar uma tendência mais conservadora no que diz respeito aos códigos de conduta das relações interpessoais.

O terceiro e último padrão é a pouca importância atribuída à honra pelos participantes de denominação espírita. Por enfatizar a evolução do espírito, participantes desta doutrina dão pouca importância a papéis tradicionais, tanto sociais, no caso da família, como de gênero (Kardec, 1996).

Pesquisas futuras sugerem a possibilidade de aprofundar a concepção de honra para estes grupos religiosos, utilizando métodos diferenciados de coleta e análise de dados, de forma a explorar a existência de dimensões locais de honra não incluídas na teoria utilizada nestes estudos. Além disso, não foram tratadas, nesta oportunidade, variáveis que podem adequadamente representar e definir a cultura, como os valores humanos (Gouveia et al., 2002). Embora se advogue a contribuição heurística do presente artigo, reconhece-se que muito há ainda que pesquisar para conhecer os antecedentes e consequentes da honra no Brasil.

\section{Agradecimento}

O presente artigo contou com apoio do $C N P q$ por meio de bolsa de Produtividade em Pesquisa concedida ao primeiro autor. Também contou com o apoio da CAPES, através de bolsa de Doutorado Pleno no exterior concedida à segunda autora. Os autores agradecem a estas instituições.

\section{Referências}

Alencar, H. \& La Taille, Y. (2007). Humilhação: O desrespeito no rebaixamento moral. Arquivos Brasileiros de Psicologia, 59, 217-231.

Baer, F. (2001). Creative proverbs from around the world. Acesso em 15 de março, 2010, em http://creativeproverbs. com/

Brannick, M. T. (2007). Corrs - MS Excel program. Acesso em 08 de dezembro, 2010, em http://luna.cas.usf.edu/ mbrannic/ files/software/softdir.htm. 
Bucher, A. (2007). Religiosity and spirituality among young adults. Bertelsmann Stiftung Foundation. Acesso em 24 de julho, 2007, em http://www.bertelsmann-stiftung.de/cps/ rde/xchg/SID-0A000F0A-11B62214/bst engl/hs.xsl/36787. $\underline{\mathrm{htm}}$.

Cohen, A. \& Hill, P. (2007). Religion as culture: Religious individualism and collectivism among American Catholics, Jews, and Protestants. Journal of Personality, 75, 709-742.

Ferrari, I. (2007). Realidade social: a violência, a segregação e a falta de vergonha. Revista Mal Estar e Subjetividade, 7, 269-284.

Fischer, A. H., Manstead, A. S. R., \& Rodriguez-Mosquera, P. M. (1999). The role of honour-related vs. individualistic values in conceptualising pride, shame, and anger: Spanish and Dutch cultural prototypes. Cognition and Emotion, 13(2), 149-179.

Geyer, A. L. \& Baumeister, R. F. (2005). Religion, morality, and self-control: Values, virtues, and vices. In R. F. Paloutzian \& C. L. Park (Eds.), Handbook of the psychology of religion and spirituality (pp. 412-432). New York: Guilford Press.

Gouveia, V. V., Albuquerque, F. J. B., Clemente, M., \& Espinosa, P. (2002). Human values and social identities: A study in two collectivist cultures. International Journal of Psychology, 37, 333-342.

Gouveia, V. \& Clemente, M. (2000). O individualismocoletivismo no Brasil e na Espanha: Correlatos sóciodemográficos. Estudos de Psicologia, 5, 317-346.

Gouveia, V.V. \& Ros, M. (2000). The Hofstede and Schwartz models for classifying individualism at the cultural level: Their relation to macro-social and macro-economic variables. Psicothema, 12, 25-33.

Guerra, V. M., Gouveia, V. V., Araújo, R. C. R., Andrade, J. M., $\&$ Bezerra, C. W. A. G. (no prelo). Honor Scale: Evidences on construct validity. Journal of Applied Social Psychology.

Hofstede, G., Hilal, A. V. G., Malvezzi, S., Tanure, B., \& Vinken, H. (2010). Comparing regional cultures within a country: Lessons from Brazil. Journal of Cross-Cultural Psychology, 41, 336-352.

Houaiss, A. (s.d.). Honra. In Dicionário Houaiss da Língua Portuguesa. Acesso em 25 de março, 2009, em http:// houaiss.uol.com.br/busca.jhtm?verbete=honra\&stype $=\mathrm{k}$.

Index Psi. (2010). Honra / reputação. Acesso em 23 de março, 2009, em http://www.bvs-psi.org.br/.

Jacob, C. R. (2004). A diversificação religiosa. Estudos Avançados, 18, 9-11.

Kardec, A. (1996). O livro dos espiritos. Brasília, DF: Federação Espírita Brasileira.

Menandro, P., Rolke, R., \& Bertollo, M. (2005). Concepções sobre relações amorosas / conjugais e sobre seus protagonistas: um estudo com provérbios. Psicologia Clínica, 17, 81-100.

Negrão, L. N. (2008). Pluralismo e multiplicidades religiosas no Brasil contemporâneo. Sociedade e Estado, 23(2), 261-279.

Nisbett, R. \& Cohen, D. (1996). Culture of honor: The psychology of violence in the South. Boulder, CO: Westview Press.

Pitt-Rivers, J. (1965). Honour and social status. In J. G. Peristiany (Ed.), Honour and shame: The values of Mediterranean society (pp. 18-77). London: Weidenfeld \& Nicholson.

Rodriguez-Mosquera, P., Fischer, A., \& Manstead, A. (2004). Inside the heart of emotion: On culture and relational concerns. In L. Z. Tiedens \& C. W. Leach (Eds.), The social life of emotions (pp. 187-202). Cambridge: Cambridge University Press.
Rodriguez-Mosquera, P., Manstead, A., \& Fischer, A. (2000). The role of honor-related values in the elicitation, experience and communication of pride, shame, and anger: Spain and the Netherlands compared. Personality and Social Psychology Bulletin, 26, 833-844.

Rodriguez-Mosquera, P., Manstead, A., \& Fischer, A. (2002a). The role of honour concerns in emotional reactions to offences. Cognition and Emotion, 16, 143-163.

Rodriguez-Mosquera, P., Manstead, A., \& Fischer, A. (2002b). Honor in the Mediterranean and Northern Europe. Journal of Cross-Cultural Psychology, 33, 16-36.

Rohden, F. (2001). Honra no Brasil: da moral sexual à imagem da nação. História, Ciências, Saúde, 3, 767-773.

Schopenhauer, A. (2003). A arte de se fazer respeitar, ou Tratado sobre a honra. São Paulo: Martins Fontes.

Schwartz, S. H. \& Rubel, T. (2005). Sex differences in value priorities: Cross-cultural and multi-method studies. Journal of Personality and Social Psychology, 89, 1010-1028.

Shackelford, T. K. (2005). An evolutionary psychological perspective on cultures of honor. Evolutionary Psychology, 3, 381-391.

Sherkat, D. \& Ellison, C. (1999). Recent developments and current controversies in the sociology of religion. Annual Review of Sociology, 25, 363-394.

Sigaud, L. (2004). Armadilhas da honra e do perdão: usos sociais do direito na mata pernambucana. Maná, 10, 131163.

Souza, M. G. T. C. (2010). Processos psicológicos do homicídio. Dissertação de Mestrado, Departamento de Psicologia, Universidade Federal de Pernambuco, Recife, PE.

Truett, K. (1993). Age differences in conservatism. Personality and Individual Differences, 14(3), 405-411.

Vandello, J. A. \& Cohen, D. (2003). Male honor and female fidelity: Implicit cultural scripts that perpetuate domestic violence. Journal of Personality and Social Psychology, 84(5), 997-1010.

Vellasco, I. (2005). A cultura da violência: Os crimes da comarca do Rio das Mortes - Minas Gerais, século XIX. Tempo, 9, 171-198.

Verztman, J. (2005). Vergonha, honra e contemporaneidade. Pulsional Revista de Psicanálise, 18, 88-99.

Werbner, P. (2007). Veiled interventions in pure space: Honour, shame and embodied struggles among Muslims in Britain and France. Theory, Culture \& Society, 24, 161-186.

Wikan, U. (1984). Shame and honour: A contestable pair. Man, $19,635-652$.

Recebido em: 06/06/201

Revisão em: 22/02/ 2012

Aceite em: 16/05/2012

Valdiney V. Gouveia é Doutor em Psicologia Social (Universidad Complutense de Madrid). Professor Titular (Universidade Federal da Paraíba). Pesquisador 1A do CNPq. Endereço: Universidade Federal da Paraíba, CCHLA - Departamento de Psicologia - João Pessoa/PB, Brasil. CEP 58051-900.

Email: vvgouveia@gmail.com

Valeschka M. Guerra é Doutora em Psicologia pela University of Kent. Professora Adjunta II da Universidade Federal do Espírito Santo - UFES. Email: valeschka@gmail.com 
Rafaella de Carvalho Rodrigues Araújo. Doutoranda em Psicologia Social na Universidade Federal da Paraíba. Email: rafaellacra@gmail.com

Lilian Kelly de Sousa Galvão. Doutora em Psicologia Social pela Universidade Federal da Paraíba. Professora Adjunta I da Universidade Federal de Campina Grande. Email: liliangalvao@yahoo.com.br

Sandra Souza da Silva. Doutora em Psicologia Social pela Universidade Federal do Rio Grande do Norte.
Professora Adjunta II da Universidade Federal da Paraíba. Email: sandra.souza psi@yahoo.com.br

\section{Como citar:}

Gouveia, V. V., Guerra, V. M., Araújo, R. C. R., Galvão, L. K. S., \& Silva, S. S. (2013). Preocupação com a honra no nordeste brasileiro: correlatos demográficos. Psicologia \& Sociedade, 25(3), 581-591. 